\title{
Average hard X-ray emission from NS LMXBs: observational evidence of different spectral states in NS LMXBs ${ }^{\star}$
}

\author{
A. Paizis ${ }^{1}$, R. Farinelli ${ }^{2}$, L. Titarchuk ${ }^{2,3,4}$, T. J.-L. Courvoisier ${ }^{5,6}$, A. Bazzano ${ }^{7}$, V. Beckmann ${ }^{3,8}$, F. Frontera $^{2}$, \\ P. Goldoni ${ }^{9}, 10$, E. Kuulkers ${ }^{11}$, S. Mereghetti ${ }^{1}$, J. Rodriguez ${ }^{10}$, and O. Vilhu ${ }^{12}$ \\ 1 INAF-IASF, Sezione di Milano, via Bassini 15, 20133 Milano, Italy \\ e-mail: ada@iasf-milano.inaf.it \\ 2 Dipartimento di Fisica, Università di Ferrara, via Saragat 1, 44100 Ferrara, Italy \\ 3 NASA Goddard Space Flight Center, Exploration of the Universe Division, Greenbelt, MD 20771, USA \\ 4 George Mason University/Center for Earth Observing and Space Research, Fairfax, VA 22030; and US Naval Research Laboratory, \\ Code 7655, Washington, DC 20375-5352, USA \\ 5 INTEGRAL Science Data Centre, Chemin d'Ecogia 16, 1290 Versoix, Switzerland \\ 6 Observatoire de Genève, 51 chemin des Mailletes, 1290 Sauverny, Switzerland \\ 7 INAF-IASF, Sezione di Roma, via del Fosso del Cavaliere 100, 00133 Roma, Italy \\ 8 Joint Center for Astrophysics, Department of Physics, University of Maryland, Baltimore County, MD 21250, USA \\ 9 APC/UMR 7164, 11 Place M. Berthelot, 75231 Paris, France \\ ${ }^{10}$ CEA, Centre de Saclay, DSM/DAPNIA/SAp, 91191 Gif-sur-Yvette Cedex, France \\ 11 ISOC, ESA/ESAC, Urb. Villafranca del Castillo, PO Box 50727, 28080 Madrid, Spain \\ 12 Observatory, PO Box 14, Tähtitorninmäki, 00014 University of Helsinki, Finland
}

Received 9 June 2006 / Accepted 10 August 2006

\section{ABSTRACT}

\begin{abstract}
Aims. We studied and compared the long-term average hard X-ray $(>20 \mathrm{keV})$ spectra of a sample of twelve bright low-mass X-ray binaries hosting a neutron star (NS). Our sample comprises the six well studied Galactic Z sources and six Atoll sources, four of which are bright ("GX") bulge sources while two are weaker ones in the 2-10 keV range (H 1750-440 and H 1608-55).

Methods. For all the sources of our sample, we analysed available public data and extracted average spectra from the IBIS/ISGRI detector on board INTEGRAL.

Results. We can describe all the spectral states in terms of the bulk motion Comptonisation scenario. We find evidence that bulk motion is always present, its strength is related to the accretion rate and it is suppressed only in the presence of high local luminosity. The two low-dim Atoll source spectra are dominated by photons up-scattered presumably due to dynamical and thermal Comptonisation in an optically thin, hot plasma. For the first time, we extend the detection of H 1750-440 up to $150 \mathrm{keV}$. The Z and bright "GX" Atoll source spectra are very similar and are dominated by Comptonised blackbody radiation of seed photons, presumably coming from the accretion disc and NS surface, in an optically thick cloud with plasma temperature in the range of $2.5-3 \mathrm{keV}$. Six sources show a hard tail in their average spectrum: Cyg X-2 (Z), GX 340+0 (Z), GX 17+2 (Z), GX 5-1 (Z), Sco X-1 (Z) and GX 13+1 (Atoll). This is the first detection of a hard tail in the X-ray spectrum of the peculiar GX $13+1$. Using radio data from the literature we find, in all Z sources and bright "GX" Atolls, a systematic positive correlation between the X-ray hard tail (40-100 keV) and the radio luminosity. This suggests that hard tails and energetic electrons causing the radio emission may have the same origin, most likely the Compton cloud located inside the NS magnetosphere.
\end{abstract}

Key words. X-rays: binaries - binaries: close - stars: neutron

\section{Introduction}

Low-Mass X-ray Binaries (LMXBs) are systems where a compact object, either a neutron star (NS) or a black hole candidate (BHC), accretes matter via Roche lobe overflow from a companion with a mass $M \lesssim 1 M_{\odot}$. NS LMXBs can be broadly classified according to their timing and spectral properties (Hasinger \& van der Klis 1989). On the basis of this classification,

* Based on observations with INTEGRAL, an ESA project with instruments and science data centre funded by ESA member states (especially the PI countries: Denmark, France, Germany, Italy, Spain, and Switzerland), Czech Republic and Poland, and with the participation of Russia and the USA.
NS LMXBs are divided in Z sources and Atoll sources from the shape of their track in the colour-colour diagram and from the different timing behaviour that correlates with the position on the tracks.

The overall spectra of $Z$ sources are very soft (Barret \& Olive 2002, and references therein) and can be described by the sum of a cool ( $\sim 1 \mathrm{keV})$ blackbody (BB) and a Comptonised emission from an electron plasma ("corona") of a few keV. Instead, Atoll sources perform quite dramatic spectral changes: when bright, they can have soft spectra (similar to $\mathrm{Z}$ sources) but they switch to low/hard spectra at low luminosities. Titarchuk \& Shaposhnikov (2005), hereafter TS05, implemented a thorough analysis of spectral and temporal properties of the Atoll 
source $4 \mathrm{U} 1728-34$. They show that the low/hard spectra at low luminosities can be described by the sum of up-scattered spectra related to the Comptonisation of the disc and NS surface soft photons. They found that the Compton cloud electron temperature is of the order of a few tens of $\mathrm{keV}$. These spectra are very similar to the hard-state spectra of BHCs but they are softer (BHC photon index $\Gamma \sim 1.6 \pm 0.1$ vs. NS $\Gamma \sim 2.1 \pm 0.1$ ) as expected from the theory (see, for example, Titarchuk \& Fiorito 2004). TS05 found that the high luminosity state spectrum of 4 U 1728-34 consists of the sum of two pure blackbody-like spectra with colour temperatures of about $1 \mathrm{keV}$ and $2.2 \mathrm{keV}$. The softer BB component is presumably related to disc emission as the harder one is related to the NS emission. It is worth noting that TS05 also found that when the source undergoes hard-soft transition, all power spectrum (PDS) frequencies (QPO and break frequencies) increase with the photon index, with no sign of saturation. Note that the index-QPO correlation observed in BHC shows the index saturation at high values of QPO frequency (see Shaposhnikov \& Titarchuk 2006).

So far, only Atoll sources (and more generally X-ray bursters) have been observed with low/hard spectra (i.e. Comptonising corona of few tens of $\mathrm{keV}$ ). Z sources always have soft Comptonisation spectra (Comptonising corona of few $\mathrm{keV}$ ) and can have an additional hard X-ray component dominating the spectrum above $\sim 30 \mathrm{keV}$. This component is on top of the soft spectrum and is highly variable with most of the emission remaining soft (see Barret 2001; Di Salvo \& Stella 2002, for a review on NS LMXB spectra). Hence, we would expect that the average high energy spectra of Atoll sources have a strong component above $30 \mathrm{keV}$ and that in the soft $\mathrm{Z}$ sources this component is less prominent and smeared out in the time averaged spectrum. $Z$ sources spend most of their time in the high/soft state, but they may show the transition to harder states at lower luminosity. The sensitivity of the past missions may have introduced an observational bias, similarly to the lack of a continuous coverage of the Galactic plane and Centre in the less explored hard X-ray range (above $20 \mathrm{keV}$ ). Moreover, the concentration of these sources towards the Galactic centre makes it difficult to observe them with non-imaging instruments. Consequently, data analysis and interpretation of such observations is extremely problematic. All these instrumental biases can be minimised with the use of the recently launched INTErnational Gamma-Ray Astrophysics Laboratory, INTEGRAL (Winkler et al. 2003). The imager INTEGRAL/IBIS (Ubertini et al. 2003) has high sensitivity, about $~ 10$ times better than GRANAT/SIGMA, coupled to imaging capability with $12^{\prime}$ angular resolution above $20 \mathrm{keV}$.

In this paper we report the study of the average hard X-ray spectra of twelve NS LMXBs performed with the low energy (20-200 keV) IBIS detector, ISGRI (Lebrun et al. 2003), using a coherent and large sample of data, free from systematic effects which play a role when combining data from different missions. The sample of the LMXBs chosen is given in Table 1 and comprises six Galactic Z sources and six Atoll sources, four of which are bright ("GX") bulge sources while two are weaker ones in the 2-10 keV range.

Our approach is two-fold: on one side, for comparison purposes, we study the average spectra in terms of phenomenological models as done in the literature, on the other, we study the sources in the frame of a physical model in the attempt to find a self-consistent scenario that describes all the spectral properties we observe. We discuss the similarities of such a scenario with the black hole LMXB case as well as the radio - X-ray correlation that is typical of LMXBs.
Table 1. LMXBs studied in this paper. $D$ : distance (in kpc) from references in Migliari \& Fender (2006) except for $(*)$ from Christian \& Swank (1997) and (**) from Ford et al. (2000); Rate: average $22-40 \mathrm{keV}$ counts/s of the source as obtained from the mosaic image shown in Fig. 1. Multiply by $\sim 10$ to obtain a flux estimate in units of mCrab. $F(1 \mathrm{mCrab})_{22-40 \mathrm{keV}} \sim 6.8 \times 10^{-12} \mathrm{erg} \mathrm{s}^{-1} \mathrm{~cm}^{-2}$; SNR: signal to noise ratio in the $22-40 \mathrm{keV}$ band; MaxEn: maximum energy channel $(\mathrm{keV})$ with a signal to noise ratio higher than three in the average spectrum; $T_{\text {exp }}$ : effective exposure time in ks.

\begin{tabular}{cccccc}
\hline Source & $\begin{array}{c}D \\
(\mathrm{kpc})\end{array}$ & $\begin{array}{c}\text { Rate } \\
(\mathrm{cps})\end{array}$ & SNR & $\begin{array}{c}\text { MaxEn } \\
(\mathrm{keV})\end{array}$ & $\begin{array}{c}T_{\exp } \\
(\mathrm{ks})\end{array}$ \\
\hline Z sources & & & & & \\
\hline Sco X-1 & 2.8 & 58.3 & 1757 & 150 & 266 \\
GX 340+0 & 11 & 2.4 & 124 & 46 & 433 \\
GX 349+2 & 5 & 3.4 & 189 & 39 & 265 \\
GX 5-1 & 9.2 & 3.8 & 330 & 80 & 1091 \\
GX 17+2 & 14 & 4.4 & 203 & 80 & 248 \\
Cyg X-2 & 13.3 & 2.2 & 57 & 55 & 149 \\
\hline Atoll sources & & & & & \\
\hline H 1608-522 & $4^{* *}$ & 0.8 & 36 & 150 & 299 \\
H 1705-440 & $11^{* *}$ & 3 & 144 & 150 & 307 \\
GX 9+9 & $5 *$ & 0.97 & 55 & 35 & 139 \\
GX 3+1 & $5.6^{*}$ & 0.96 & 86 & 43 & 2027 \\
GX 9+1 & $7 *$ & 1.2 & 91 & 37 & 491 \\
GX 13+1 & 7 & 0.96 & 57 & 80 & 290 \\
\hline
\end{tabular}

\section{Observations and data analysis}

We have analysed INTEGRAL data publicly available in which the sources in Table 1 were in the Fully Coded Field of View (FCFOV) of IBIS. The additional criterion of a minimum of good time of $1000 \mathrm{~s}$ for IBIS/ISGRI led to a total of 2263 pointings each with variable exposure time (from about $1800 \mathrm{~s}$ up to about 3600 s) spanning from January 2003 to May 2004. Version 5.1 of the Off-line Scientific Analysis (OSA) software has been used to analyse the data. The description of the algorithms used in the IBIS/ISGRI scientific analysis can be found in Goldwurm et al. (2003).

For each pointing we extracted images in the 22-40 and $40-80 \mathrm{keV}$ energy bands. The images were used to build lightcurves as well as a final mosaic. The mosaic images revealed the sources that were active in the field of view at the time of the observations. The list of all the detected sources (one per source of interest) was then used in the spectral step, where spectra for all the active sources in the field of view were simultaneously extracted with the standard spectral extraction method. The single pointing spectra were then averaged into one final spectrum per source. These final average spectra have been used in our spectral study adding $1 \%$ systematic error (with the exception of the bright Sco $\mathrm{X}-1$ for which we added $1.5 \%$ systematic error). In the fit, only data points with more than $3 \sigma$ in the $22-200 \mathrm{keV}$ range have been considered.

To cross-check our results, we also used the alternative method of extracting spectra from the mosaics. To do so, we re-ran the imaging step in twelve different bins (instead of the 22-40 and 40-80 keV previously mentioned) for all the pointings and then extracted a spectrum using the flux from each energy map. We verified that the two different methods give compatible results. In this work we show only the results of the former method (the standard extraction) for which we have a finer binning. 


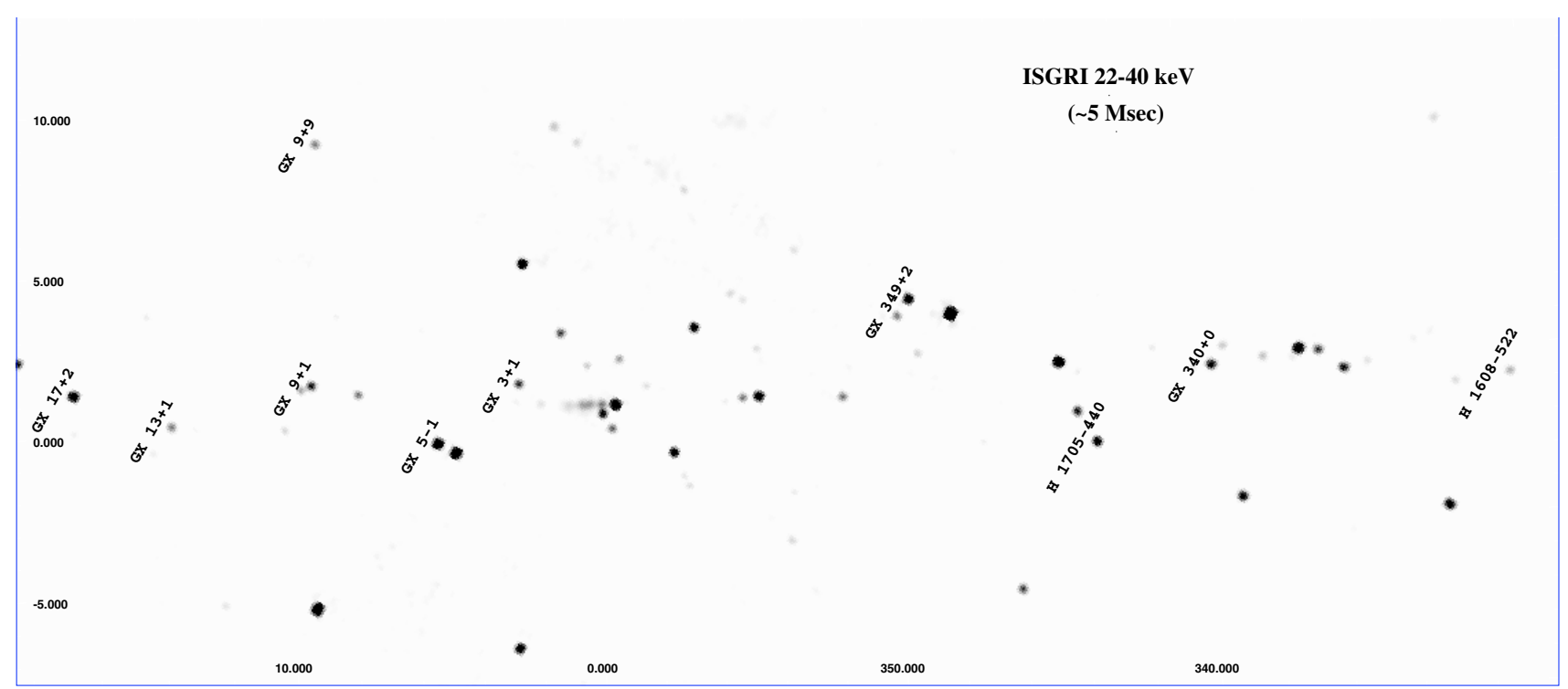

Fig. 1. IBIS/ISGRI 22-40 keV mosaic of the Galactic bulge (about $5 \mathrm{Ms}$ ). Only the sources studied in this paper are labeled. The location of Cyg $\mathrm{X}-2$ and Sco $\mathrm{X}-1$ is not covered by this map.

\section{Results}

Figure 1 shows the final sky map of the pointings around the Galactic bulge in the $22-40 \mathrm{keV}$ band. The sources studied in this paper are labeled, with the exception of Cyg X-2 and Sco $\mathrm{X}-1$ that lie outside the image. The $22-40 \mathrm{keV}$ average count rate and detection significance of each source, taken from the mosaic image shown in Fig. 1, are reported in Table 1. The count rate is in counts/s and multiplied by $\sim 10$ gives an estimate in $\mathrm{mCrab}^{1}$.

Figures 2 and 3 show the IBIS/ISGRI (22-40 keV and $40-80 \mathrm{keV}$ ) light-curves as obtained from the imaging analysis (re-binned to ten day data bins). The simultaneous quick-look results provided by the RXTE/ASM team are also shown (one day data bins). One Crab is approximately 75 counts/s in the RXTE/ASM 2-12 keV energy band, 93 counts/s and 61 counts/s in the IBIS/ISGRI 22-40 and 40-80 keV band, respectively.

\subsection{A phenomenological approach}

As a first attempt we tried to fit all the spectra with the same simple model, in order to compare them directly. It soon turned out that this was not possible since the long-term average spectra identified three main spectral states. Phenomenologically, we can classify them in terms of a widely used thermal Comptonisation model, COMPTT by Titarchuk (1994). We found that the spectra of GX 349+2, GX 9+1, GX 9+9, and GX 3+1 are well described by a single cOMPTT component while we need two components, COMPTT + PL (thermal Comptonisation plus power-law), to describe the data for Sco X-1, GX 5-1, GX 340+0, GX 17+2, Cyg X-2 and GX 13+1. On the other hand, the spectral shapes of $\mathrm{H} 1608-522$ and $\mathrm{H}$ 1705-440 can be well fitted by a simple power-law, PL.

By comparison with black hole LMXBs, hereafter we call the spectral states fitted with a single COMPTT a very soft state for which the spectral shape is well described by the blackbodylike shape slightly modified by Comptonisation. Using this analogy, we call our COMPTT+PL-state an intermediate state. In

1 The average Crab count rate in different positions of the FCFOV in the same band is about 93 counts/s. fact, the intermediate state in the BHCs is characterized by the blackbody-like component at low energies and the steep powerlaw component at higher energies. In the low/hard state spectra of BHCs, the blackbody signature is smeared out but the powerlaw is prominent. Keeping in mind this observational fact for the BHCs, we can call our PL state a low/hard state. The three states are shown in Fig. 4. In the plot we have also included the spectrum of the Atoll source GX 354-0 (Falanga et al. 2006) to point out that the low/hard state of our classification, associated to $\mathrm{H} 1608-522$ in the plot, is not a "pure" low/hard state as in the case of GX 354-0 (thermal Comptonisation with cut-off) but is a low/hard state where an additional physical mechanism producing a non-attenuated power-law starts to be important (see Sect. 4.1). Nevertheless, for simplicity hereafter we will refer to the spectra of H 1608-522 and H 1705-440 as to low/hard states. Table 2 summarises the spectral models used to fit the data while the corresponding plots with the spectra and best fit models are shown in Figs. 5 and 6.

\subsubsection{The very soft spectral state}

Three bright Atoll sources (GX 3+1, GX 9+1 and GX 9+9) and the $\mathrm{Z}$ source GX $349+2$ can be well described by a simple COMPTT component. A hard tail has been observed to dominate the spectra above $30 \mathrm{keV}$ in a pointed observation of $\mathbf{G X ~ 3 4 9 + 2}$ (Di Salvo et al. 2001), but it is likely not frequent/bright enough to be detected in a $265 \mathrm{ks}$ average spectrum. Indeed, no hard tail was detected in the spectrum of GX $349+2$ by Iaria et al. (2004) using BeppoSAX data even though the source was in the same position in the Z-track where the tail was first discovered. No hard tails have ever been detected in the spectra of GX 9+9, GX $9+1$ and GX 3+1.

Only recent results have appeared as far as the X-ray spectrum of GX 9+9 is concerned. Kong et al. (2006), based on $R X T E$ data, report a spectrum that can be described by the typical two component model used for bright LMXBs hosting a neutron star: a softer component (e.g. blackbody) plus a Comptonised harder one (e.g. COMPTT). We are not sensitive to the soft blackbody component with IBIS/ISGRI and our results as far as the COMPTT part are concerned are in agreement 

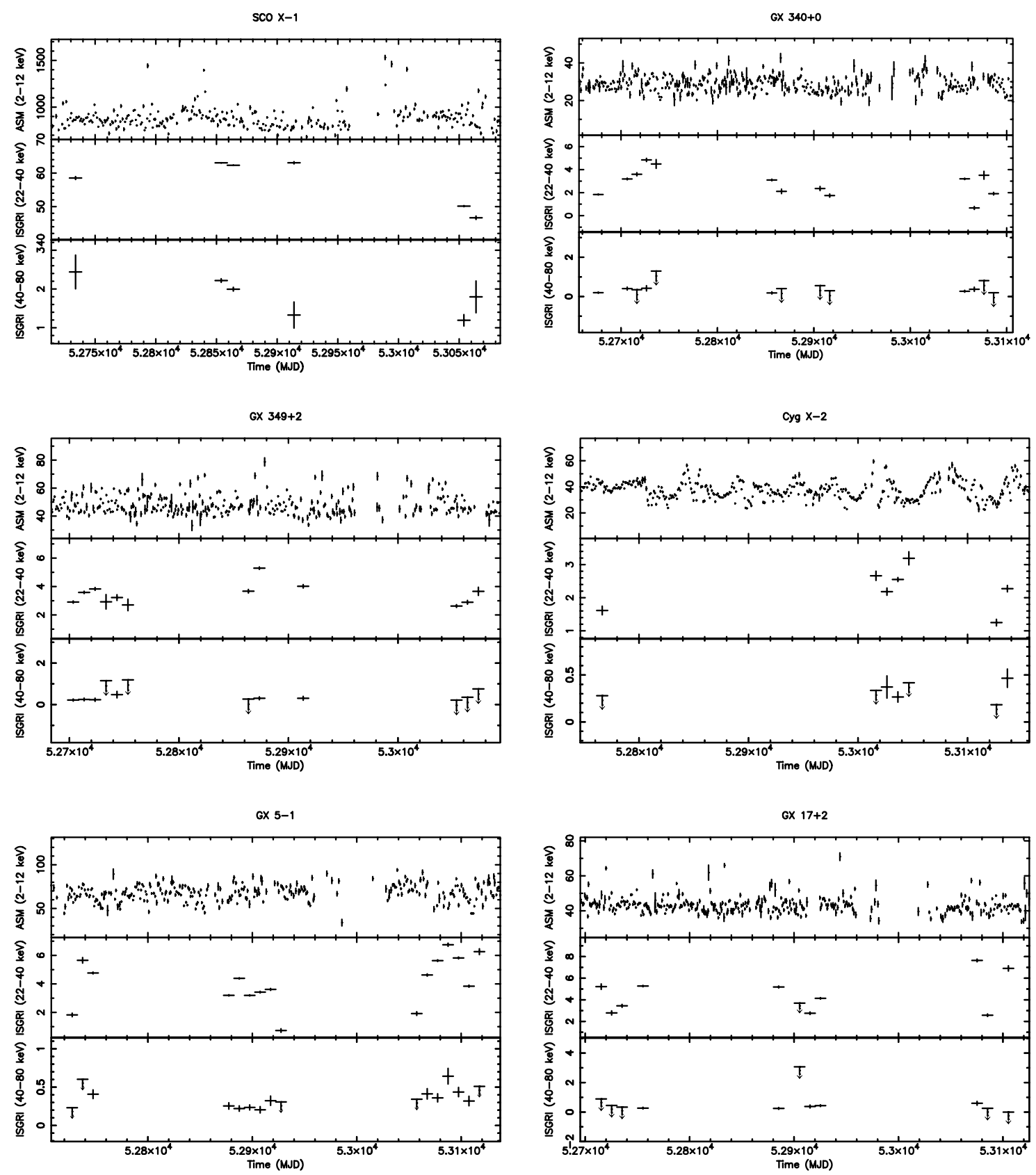

Fig. 2. RXTE/ASM and IBIS/ISGRI light-curves (counts/s) of the $\mathrm{Z}$ sources. One Crab is approximately 75 counts/s in RXTE/ASM (2-12 keV), 93 counts/s in IBIS/ISGRI $22-40 \mathrm{keV}$ and 61 counts/s in IBIS/ISGRI $40-80 \mathrm{keV}$.

with what was found by Kong et al. (2006) i.e. a Comptonising plasma $k T_{\mathrm{e}}$ of about $4 \mathrm{keV}$ with a high optical depth $\tau$ about 7 . Our RXTE-INTEGRAL simultaneous observations (Vilhu et al. 2006) show that the broad band spectrum of GX $9+9$ can be also well described by the sum of a soft component (DISKBB in XSPEC terminology) and thermal Comptonisation (COMPBB) by a hot optically thin electron plasma.

The fit we obtain for GX 3+1 is not $\operatorname{good}\left(\chi^{2} /\right.$ d.o.f. $\left.=46 / 24\right)$; however, as can be seen in Fig. 6, this is not due to a clear excess or systematics, more likely it is due to source spectral variability. Our average spectrum parameters show that also GX $3+1$ has a Z-like spectrum, similarly to what found by Oosterbroek et al. (2001) using BeppoSAX data (optical depth $\tau$ about 6 and Comptonising plasma temperature $k T_{\mathrm{e}}$ about $2.7 \mathrm{keV}$ ).

Iaria et al. (2005) presented the $0.12-18 \mathrm{keV}$ spectrum of GX 9+1 from a long BeppoSAX observation. The $0.12-18 \mathrm{keV}$ spectrum could be well fitted by a blackbody model plus Comptonised component, the latter with an electron temperature $k T_{\mathrm{e}}$ of $2.3 \mathrm{keV}$ and optical depth $\tau$ of about 15 . No firm conclusion could be drawn above $20 \mathrm{keV}$ due to source confusion issues. The INTEGRAL observation above $20 \mathrm{keV}$ does not suffer from source confusion and shows that the spectrum of GX 9+1 is very similar to the bright Atoll sources GX 9+9 and GX 3+1, i.e. very similar to the typical $Z$ source spectrum. There is a hint of excess in the spectrum of GX 9+1, Fig. 6, but it is not significant with the current exposure time.

\subsubsection{The intermediate spectral state}

A simple COMPTT model was not good enough to describe the data due to a systematic excess above $\sim 30 \mathrm{keV}$ for Sco X-1 

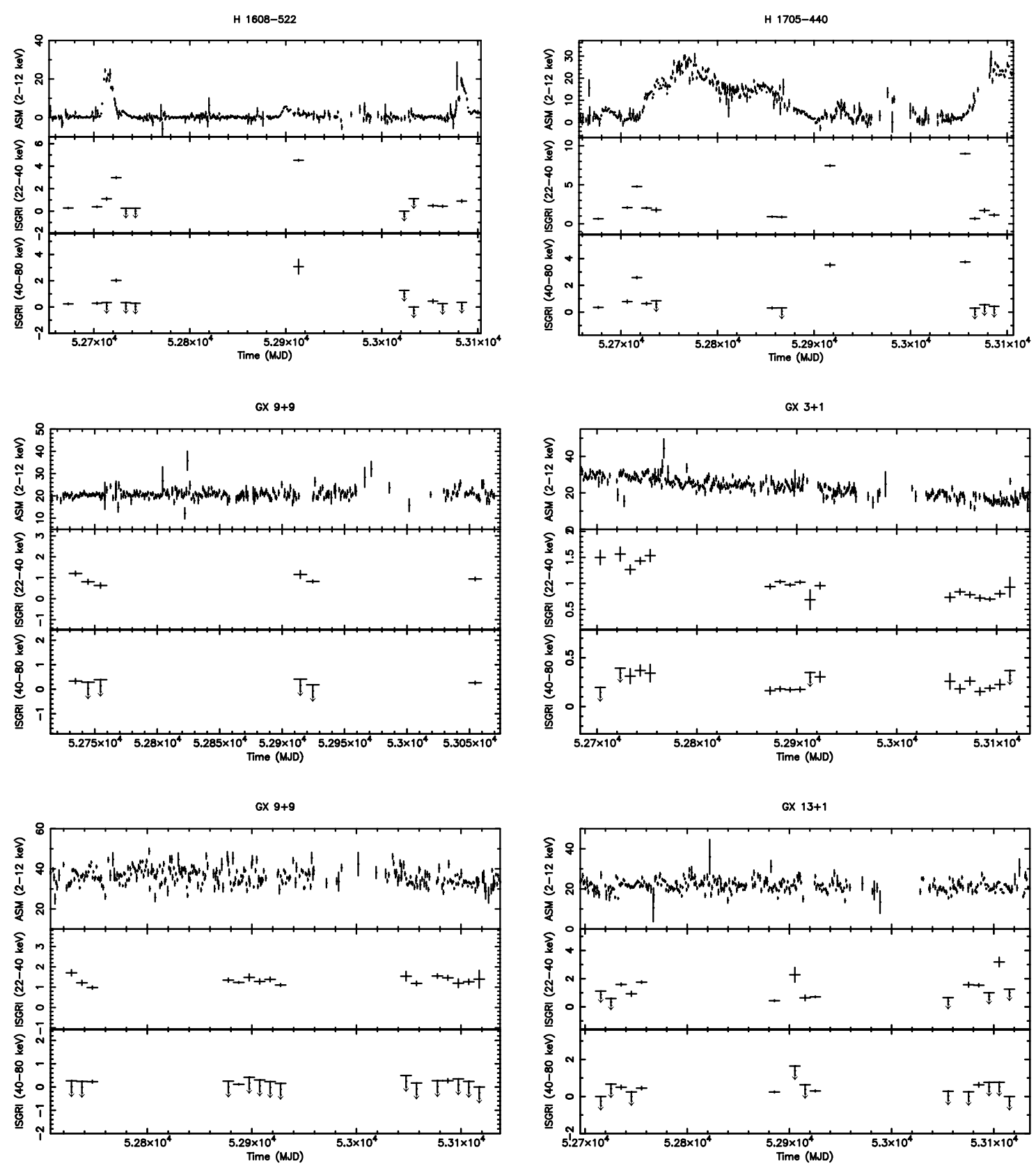

Fig. 3. RXTE/ASM and IBIS/ISGRI light-curves (counts/s) of the Atoll sources. One Crab is approximately 75 counts/s in RXTE/ASM (2-12 keV), 93 counts/s in IBIS/ISGRI 22-40 keV and 61 counts/s in IBIS/ISGRI 40-80 keV.

$\left(\chi^{2} /\right.$ d.o.f. $\left.>60\right)$, Cyg X-2 (45/27), GX 17+2 (61/31), GX 5-1 (96/31), and GX 13+1 (38/24). With the addition of a power-law, the fits improved significantly (see Table 2) with the temperature of the Comptonising plasma, $k T_{\mathrm{e}}$, stabilising itself around 2.5-3 keV. Within this sample, transient hard tails had already been observed in the spectra of Sco X-1 (D'Amico et al. 2001) based on RXTE data, GX 5-1 (Paizis et al. 2005) based on INTEGRAL data, GX 17+2 (Farinelli et al. 2005) and Cyg X-2 (Di Salvo et al. 2002) based on BeppoSAX data. In our study, the seed photon temperature could not be constrained and was fixed to the value [1.0-1.3] keV for all the sources, with the exception of GX $340+0$ for which it was fixed at $0.93 \mathrm{keV}$ (Lavagetto et al. 2004), and GX 17+2 fixed at $0.6 \mathrm{keV}$ (Farinelli et al. 2005).

The spectrum of $\mathbf{G X} \mathbf{3 4 0 + 0}$ could be fitted with a simple COMPTT model $\left(\chi^{2} /\right.$ d.o.f. $\left.=32 / 29\right)$ with $k T_{\text {seed }}$ fixed at $0.93 \mathrm{keV}$ as found by Lavagetto et al. (2004), providing $k T_{\mathrm{e}}=6.5_{-2.1}^{+6.7} \mathrm{keV}$ and $\tau=1.7_{-1.2}^{+2.8}$. We note however, that if we freeze $k T_{\mathrm{e}}$ to $3 \mathrm{keV}$, as found in the BeppoSAX broad-band fit $(0.1-100 \mathrm{keV})$ by Lavagetto et al. (2004), around $\sim 40 \mathrm{keV}$ the residuals systematically deviate from COMPTT and the fit is no longer acceptable $\left(\chi^{2} /\right.$ d.o.f. $\left.=58 / 27\right)$. In this case the addition of a power-law ( $\Gamma$ frozen at 2.5, as found in BeppoSAX data) is required.

The Comptonising plasma parameters obtained in the fit of the spectrum of Sco $\mathbf{X}-\mathbf{1}$ show values typical of $\mathrm{Z}$ sources $\left(k T_{\mathrm{e}}\right.$ of a few $\mathrm{keV}$ and optical depth between 5-15). The excess left by this model is well fitted by a power-law with no measured cut-off up to $150 \mathrm{keV}$. Such an extended power-law can be an indication for bulk motion (dynamical) Comptonisation or non-thermal electron population given the bad fit obtained with different thermal Comptonisation models. See Di Salvo et al. (2006) for a study of the spectral variability of Sco X-1 with INTEGRAL. 
Table 2. Summary of the spectral properties of the sources. No error means the parameter was fixed to the indicated value. Model: best fit model. See text for details; $\Gamma$ : photon index of the power-law; $k T_{\text {seed }}$ : temperature of seed photons for comptonisation in keV; $k T_{\mathrm{e}}$ : Comptonising plasma temperature in $\mathrm{keV} ; \tau$ : optical depth of the Comptonising plasma; Flux $(20-40 \mathrm{keV})$ : flux obtained between $20-40 \mathrm{keV}$. For the sources detected up to $150 \mathrm{keV}$ also the $40-150 \mathrm{keV}$ flux is given.

\begin{tabular}{ccccccccc}
\hline \hline Source & Model & $\Gamma$ & $\begin{array}{c}k T_{\text {seed }} \\
(\mathrm{keV})\end{array}$ & $\begin{array}{c}k T_{\mathrm{e}} \\
(\mathrm{keV})\end{array}$ & $\tau$ & $\chi^{2} /$ d.o.f. & $\begin{array}{c}\text { Flux }(20-40 \mathrm{keV}) \\
\left(\mathrm{erg} \mathrm{s}^{-1} \mathrm{~cm}^{-2}\right)\end{array}$ & $\begin{array}{c}\text { Flux } \\
(40-150 \mathrm{keV}) \\
\left(\mathrm{erg} \mathrm{s}^{-1} \mathrm{~cm}^{-2}\right)\end{array}$ \\
\hline Sco X-1 & COMPTT+PL & $3.85_{-0.08}^{+0.01}$ & {$[1.3]$} & $2.87_{-0.02}^{+0.01}$ & $9.45_{-0.34}^{+0.11}$ & $42 / 34$ & $5.8 \times 10^{-9}$ & $2.2 \times 10^{-10}$ \\
GX 340+0 & COMPTT+PL & {$[2.5]$} & {$[0.93]$} & {$[3.0]$} & $7.73_{-0.78}^{+0.93}$ & $24 / 26$ & $2.4 \times 10^{-10}$ & \\
GX 349+2 & COMPTT & - & {$[1.3]$} & $2.75_{-0.15}^{+0.33}$ & $12_{-5}^{+16}$ & $22 / 24$ & $3.5 \times 10^{-10}$ & \\
GX 5-1 & COMPTT+PL & $4.7_{-0.11}^{+0.6}$ & {$[1.0]$} & $2.42_{-0.06}^{+0.41}$ & $25_{-16}^{+5}$ & $35 / 29$ & $3.5 \times 10^{-10}$ & \\
GX 17+2 & COMPTT+PL & {$[2.7]$} & {$[0.6]$} & $2.94 \pm 0.04$ & {$[11]$} & $35 / 31$ & $4.5 \times 10^{-10}$ & \\
Cyg X-2 & COMPTT+PL & {$[1.96]$} & {$[1.09]$} & $3.02_{-0.10}^{+0.10}$ & 10.68 & $29 / 27$ & $2.1 \times 10^{-10}$ & \\
\hline H 1608-522 & PL & $2.62 \pm 0.11$ & - & - & - & $45 / 36$ & $8.7 \times 10^{-11}$ & $9.1 \times 10^{-11}$ \\
& COMPPS & - & $2.29_{-0.25}^{+1.14}$ & $122_{-43}^{+24}$ & $0.18_{-0.13}^{+0.43}$ & $40 / 34$ & & \\
H 1705-440 & PL & $3.15 \pm 0.04$ & - & - & - & $36 / 37$ & $3 \times 10^{-10}$ & $1.9 \times 10^{-10}$ \\
& COMPPS & - & $2.56_{-0.47}^{+0.62}$ & $47 \pm 7$ & $0.63_{-0.17}^{+0.18}$ & $31 / 35$ & & \\
GX 9+9 & COMPTT & - & {$[1.3]$} & $3.66_{-1.00}^{+25}$ & $6(>0.4)$ & $27 / 23$ & $9.2 \times 10^{-11}$ & \\
GX 3+1 & COMPTT & - & {$[1.3]$} & $2.88_{-0.28}^{+0.59}$ & $7.5_{-2.9}^{+4.7}$ & $46 / 24$ & $9.3 \times 10^{-11}$ & \\
GX 9+1 & COMPTT & - & {$[1.3]$} & $2.30_{-0.08}^{+0.45}$ & $26(>7)$ & $32 / 22$ & $1.3 \times 10^{-10}$ & \\
GX 13+1 & COMPTT+PL & {$[2.8]$} & {$[1.0]$} & $2.75_{-0.23}^{+0.25}$ & {$[8]$} & $23 / 22$ & $8.2 \times 10^{-11}$ & \\
\hline
\end{tabular}

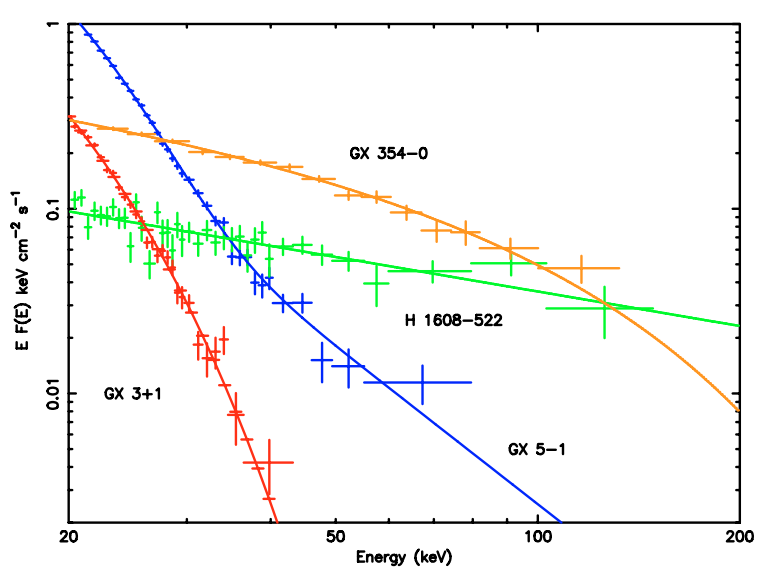

Fig. 4. IBIS/ISGRI average spectra of GX 354-0 ("pure" low/hard state, see text), H 1608-522 (low/hard state), GX 5-1 (intermediate state) and GX $3+1$ (very soft state).

Farinelli et al. (2005) used the same model used here (COMPTT plus power-law) to describe the spectrum of $\mathbf{G X} \mathbf{1 7}+\mathbf{2}$, so a more quantitative comparison is possible. We fixed the power-law slope $(\Gamma=2.7)$, optical depth $(\tau=11)$ and seed photon temperature $\left(k T_{\text {seed }}=0.6 \mathrm{keV}\right)$ to what was found by Farinelli et al. (2005) and find a similar electron gas temperature but a lower power-law normalization at $1 \mathrm{keV}$ (our $\sim 0.23$ versus their $\sim 2.1$ ). This is compatible with a picture of a fairly constant soft component and transient hard tail component that is at maximum when observed in a pointed observation (Farinelli et al. 2005) and at a lower value when averaged on long-term observations (due to its transient nature).

Similarly, for Cyg X-2 the power-law slope could not be constrained and we used the average value found by Di Salvo et al. (2002) who also used the COMPTT plus power-law model. The hard tail was the strongest in the horizontal branch and we used the photon index average value from the upper and lower horizontal branch $(\sim 2)$. The same was done for the seed photon temperature $(\sim 1 \mathrm{keV})$ and optical depth $(\sim 11)$. Similarly to GX $17+2$, we obtain an electron gas temperature close to that found by Di Salvo et al. (2002) and a power-law normalization of $\sim 0.02$ (to be compared to their $\sim 0.03$ ). The difference in the power-law normalization is much less than for the case of GX 17+2. This could be due to the fact that Cyg X-2 spends more time than GX $17+2$ on the horizontal branch and so the average spectrum of $\mathrm{Cyg} \mathrm{X}-2$ is closer to the horizontal branch spectrum than in the case of GX $17+2$.

The hard X-ray spectrum of GX 5-1 has always been difficult to study due to the presence of the nearby black hole candidate GRS 1758-258 (40' apart). Asai et al. (1994) first detected a possible hard tail in the spectrum of GX 5-1 although contamination from the nearby black hole could not be excluded. The first non-contaminated observation of a systematic hardening of the source spectrum in the horizontal branch was done by Paizis et al. (2005) with INTEGRAL data and the presence of the hard tail is confirmed here, based on a larger data set.

The addition of the power-law component in the spectrum of GX 13+1 required some parameters to be frozen. We fixed the seed photon temperature and optical depth to the values found by Homan et al. (2004) ( 1 and $8 \mathrm{keV}$, respectively). The photon index was found to be steady around 2.8 (even with a cutoff PL plus PL model and bremsstrahlung plus PL). Fixing the slope to 2.8, we obtained the Comptonising plasma temperature of about $2.75 \mathrm{keV}$ given in Table 2. The nature of GX $13+1$ has always been a matter of debate: Hasinger \& van der Klis (1989) classified it as a bright Atoll source (hence our classification), although they noted that it showed properties which were closest to those seen in the Z sources; Schulz et al. (1989) grouped it with the high luminosity sources that included the six sources that were later classified as Z sources; Homan et al. (1998) discovered quasi periodic oscillations (QPOs) similar to the horizontal branch oscillations found in $\mathrm{Z}$ sources; based on simultaneous radio and X-ray observations, Homan et al. (2004) showed that the X-ray and radio luminosities of GX 13+1 are actually in the range expected for $\mathrm{Z}$ sources unlike the Atoll sources GX 9+1, GX 9+9 and GX 3+1 that have similar X-ray luminosities but much lower radio ones. Our work shows that indeed GX $13+1$ behaves like a Z source not only due to its variability and radio properties but also from the spectral point 

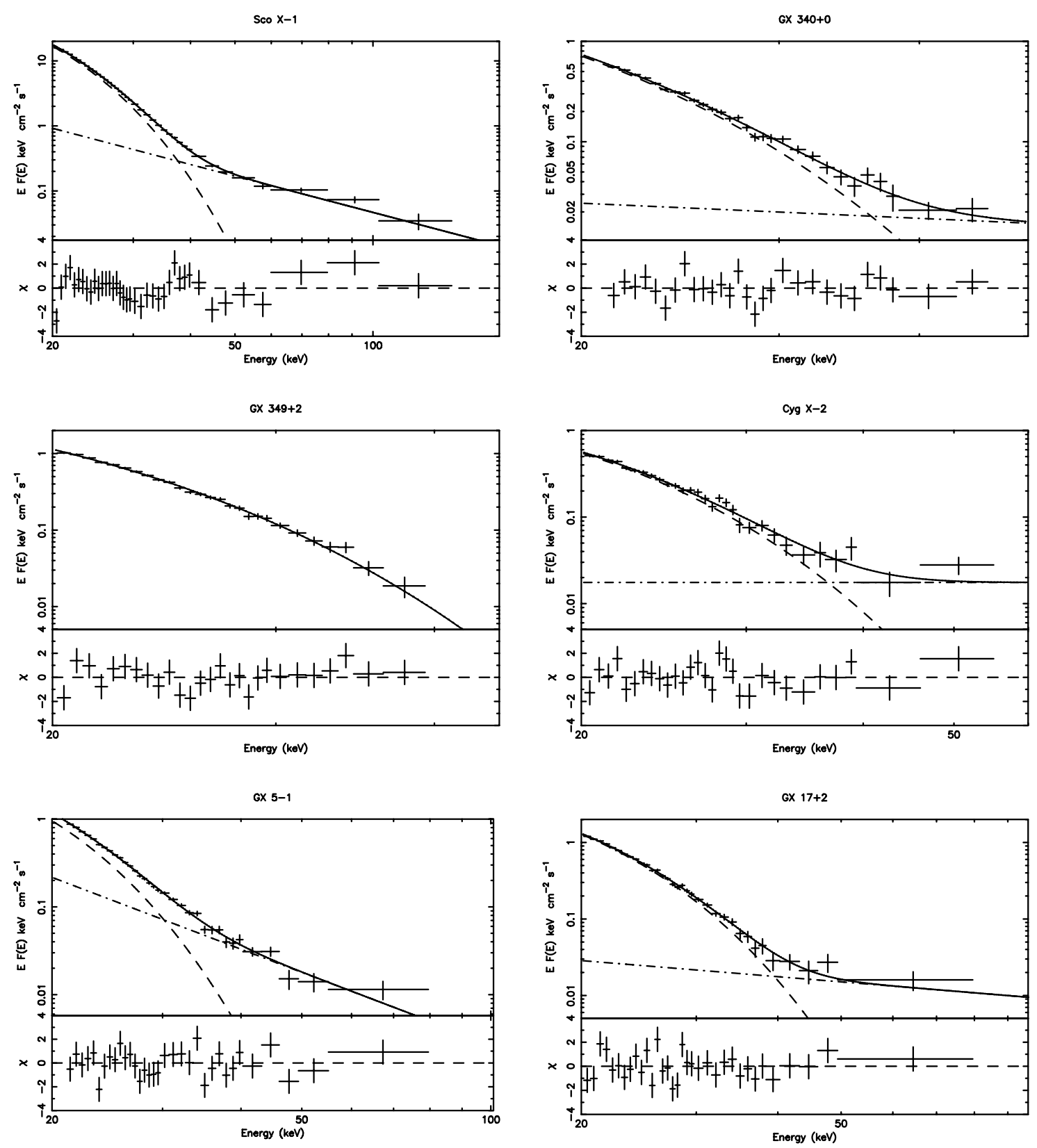

Fig. 5. Z sources: average IBIS/ISGRI spectra and best fit models shown in Table 2 .

of view with an average spectrum very similar to $\mathrm{Z}$ sources, i.e. thermal Comptonisation plus hard tail.

\subsubsection{The low/hard spectral state}

The two remaining Atoll sources, H 1608-522 and H 1705-44, are the only ones of our sample that can be well described by a single power-law. The burster $\mathbf{H ~ 1 6 0 8 - 5 2}$ has been detected beyond $100 \mathrm{keV}$ also in the past (Zhang et al. 1996, with BATSE observations) when it was in a very bright state (about $100 \mathrm{mCrab}, 20-100 \mathrm{keV}$ ) with a spectral break or cut-off around $65 \mathrm{keV}$.

H 1705-44 has been observed to show dramatic spectral changes in its X-ray spectrum (Barret \& Olive 2002). The $2-80 \mathrm{keV}$ could be well fitted by a blackbody plus Comptonisation model (COMPTT) with a temperature of about $15 \mathrm{keV}$ i.e. the spectrum was not detected above $100 \mathrm{keV}$. In this study we detect the source up to about $150 \mathrm{keV}$, that, to the best of our knowledge, is a new result. The high sensitivity of IBIS/ISGRI combined with the long observation time (307 ks) are essential ingredients for this new detection, similarly to what happened e.g. in the case of Aql X-1 (Rodriguez et al. 2006) and 4U 1850-087 (Sidoli et al. 2006).

To have a feeling of the plasma temperature in $\mathrm{H}$ 1608-522 and $\mathrm{H}$ 1705-440, we fitted their spectra with the Comptonisation model by Poutanen \& Svensson (1996) (COMPPS in XSPEC terminology) obtaining Comptonisation of soft seed photons $(\sim 2 \mathrm{keV})$ by an optically thin $(\tau<1)$ hot plasma. We assumed a spherical geometry and a blackbody seed photon spectrum (Table 2).

\subsection{Generic comptonisation model}

As we have mentioned above, in our analysis of X-ray spectra from NS LMXBs we found similarities with black hole LMXBs. The BHC and NS spectral shapes are generic: they consist of 

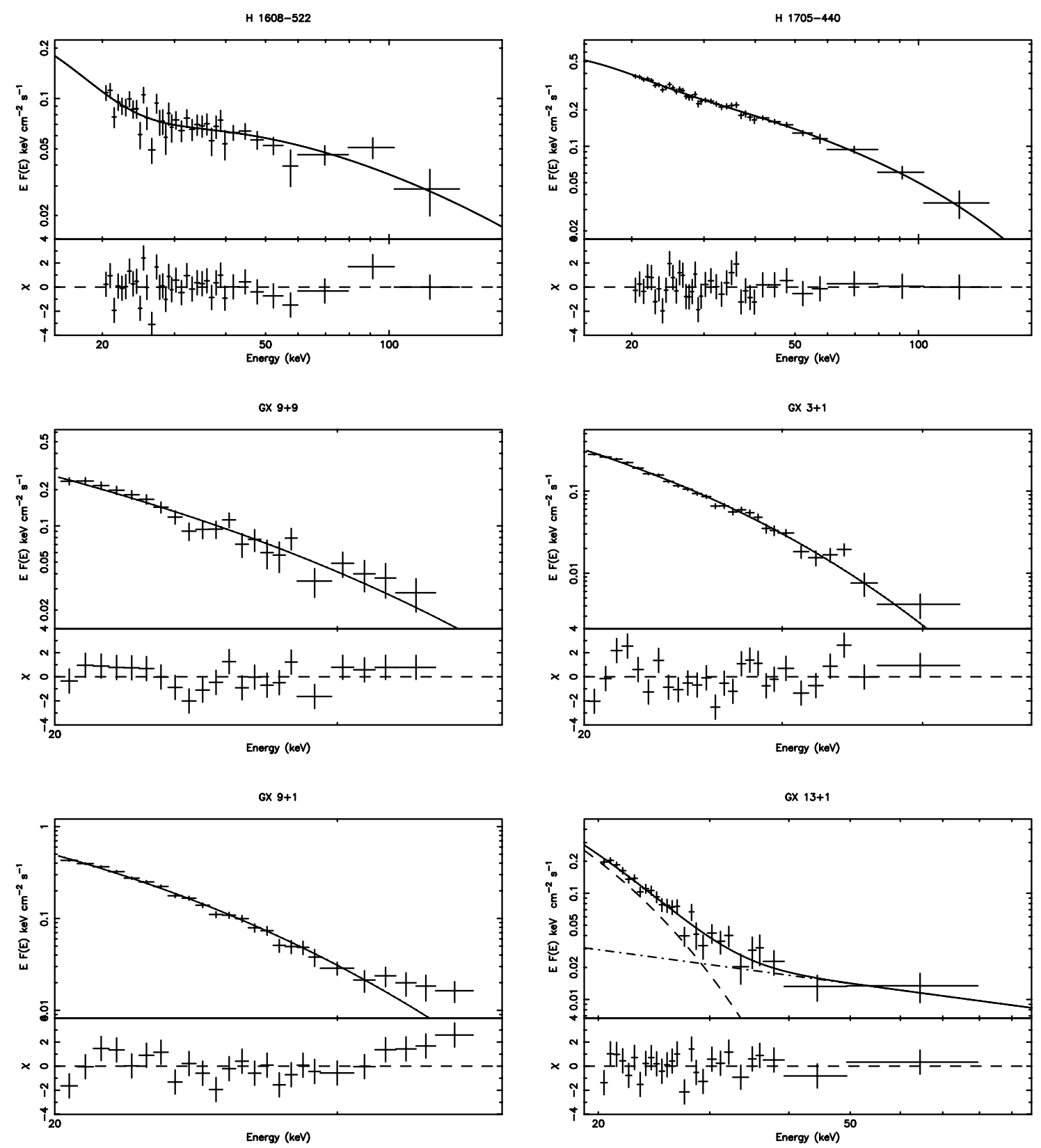

Fig. 6. Atoll sources: average IBIS/ISGRI spectra and best fit models shown in Table 2.

blackbody-like (BB) and power-law components. Sometimes one needs an exponential rollover in order to terminate the power-law component at high energies. The main difference is that NS spectra are usually softer for the same state. Another difference in these spectra is that in the NS case there are two BB components (not one as in BHC spectra) which can be related to the emission from the disc (BB colour temperature about $1 \mathrm{keV}$ ) and NS surface (BB colour temperature about $2.5 \mathrm{keV})$, (TS05).

Titarchuk et al. (1996, 1997), hereafter TMK96 and TMK97, introduced the Generic Comptonisation model (BMC model in XSPEC) that takes into account the dynamical Comptonisation (converging inflow, expected in the vicinity of the central object) along with thermal Comptonisation. It is worth noting that this model can be applied to fit the observed spectra of NS and BHC sources. The BMC model reads as:

$F(E)=\frac{C_{N}}{1+A}(B B+A \times B B * G)$ where $G\left(E, E_{0}\right)$ is the Green's function obtained as a solution of radiative diffusion equation in which both effects of thermal and dynamical Comptonisation are included. $B B$ stands for the injected blackbody-like spectrum, namely $B B \propto B_{v}\left(T_{\mathrm{col}}\right), B B * G$ is a convolution of $B B$ with the (Comptonisation) Green's function $G\left(E, E_{0}\right), C_{N}$ is the BB normalization coefficient (that depends on the compact object mass and distance to the source). The factor $1 /(1+A)$ is the fraction of the seed photon radiation directly seen by the Earth observer, whereas the factor $A /(1+A)$ is the fraction of the seed photon radiation up-scattered by the Compton cloud.

$G\left(E, E_{0}\right)$ is the response of the Compton medium (cloud) to the injection of monochromatic line with energy $E_{0}$. TMK97 showed that $G\left(E, E_{0}\right)$ is a broken power-law with blue wing index $\alpha$ and red wing index always more than $\alpha+3$. Note that $G\left(E, E_{0}\right)$ approaches Delta-function $\delta\left(E-E_{0}\right)$ for $\alpha \gg 1$. High values of $\alpha$ are an indication of low efficiency of Comptonisation that usually occurs when the plasma of the 
Table 3. Best fit parameters for the bulk motion Comptonisation model (BMC). No error means the parameter was fixed to the indicated value. $k T_{b b}$ : BB colour temperature, in keV; $\alpha$ : energy spectral index $(\Gamma=\alpha+1)$; $\log A$ : covering of the BB by the Compton cloud; Flux $(20-40 \mathrm{keV})$ : flux obtained between $20-40 \mathrm{keV}$. For the sources detected up to $150 \mathrm{keV}$ also the $40-150 \mathrm{keV}$ flux is given.

\begin{tabular}{ccccccc}
\hline \hline Source & $\begin{array}{c}k T_{\mathrm{bb}} \\
(\mathrm{keV})\end{array}$ & $\alpha$ & $\log A$ & $\chi^{2} /$ d.o.f. & $\begin{array}{c}\text { Flux }(20-40 \mathrm{keV}) \\
\left(\mathrm{erg} \mathrm{s}^{-1} \mathrm{~cm}^{-2}\right)\end{array}$ & $\begin{array}{c}\text { Flux }_{(40-150 \mathrm{keV})} \\
\left(\mathrm{erg} \mathrm{s}^{-1} \mathrm{~cm}^{-2}\right)\end{array}$ \\
\hline Sco X-1 & $2.56_{-0.01}^{+0.01}$ & $3.27_{-0.02}^{+0.06}$ & $-1.47_{-0.01}^{+0.01}$ & $68 / 35$ & $5.8 \times 10^{-9}$ & $2.2 \times 10^{-10}$ \\
GX 340+0 & $2.43_{-0.31}^{+0.18}$ & $<6$ & $-1.12_{-1.05}^{+0.81}$ & $24 / 25$ & $2.45 \times 10^{-10}$ & \\
GX 349+2 & $2.47_{-0.12}^{+0.04}$ & $<6$ & $-1.62_{-1.03}^{+0.69}$ & $20 / 23$ & $3.5 \times 10^{-10}$ & \\
GX 5-1 & $2.35_{-0.09}^{+0.07}$ & $3.84_{-0.66}^{+0.72}$ & $-1.06_{-0.28}^{+0.28}$ & $34 / 30$ & $3.75 \times 10^{-10}$ & \\
GX 17+2 & $2.68_{-0.06}^{+0.06}$ & $2.0_{-1.41}^{+1.46}$ & $-1.87_{-0.45}^{+0.55}$ & $32 / 30$ & $4.5 \times 10^{-10}$ & \\
Cyg X-2 & $2.78_{-0.07}^{+0.08}$ & $<1.8$ & $-0.56_{-0.14}^{+0.11}$ & $26 / 26$ & $2.1 \times 10^{-10}$ & \\
\hline H 1608-522 & $1.49_{-0.73}^{+1.39}$ & $1.50_{-0.16}^{+0.14}$ & $-1.24_{-2.5}^{+1.45}$ & $39 / 34$ & $8.5 \times 10^{-11}$ & $9.6 \times 10^{-11}$ \\
H 1705-440 & $0.84_{-0.84}^{+0.14}$ & $2.15_{-0.05}^{+0.05}$ & $-2.13_{-3.85}^{+10.13}$ & $36 / 35$ & $2.9 \times 10^{-10}$ & $1.9 \times 10^{-10}$ \\
GX 9+9 & $2.53_{-0.17}^{+0.18}$ & $<6$ & $-0.70_{-1.36}^{+0.34}$ & $24 / 22$ & $9.4 \times 10^{-11}$ & \\
GX 3+1 & $2.30_{-0.14}^{+0.11}$ & $<6$ & $-1.27_{-1.32}^{+0.37}$ & $42 / 23$ & $9.3 \times 10^{-11}$ & \\
GX 9+1 & $2.20_{-0.07}^{+0.05}$ & $<3.2$ & $-1.09_{-0.33}^{+0.16}$ & $24 / 21$ & $1.32 \times 10^{-10}$ & \\
GX 13+1 & $2.32_{-0.36}^{+0.27}$ & $2.02_{-1.33}^{+1.48}$ & $-1.34_{-0.50}^{+0.58}$ & $23 / 23$ & $8.2 \times 10^{-11}$ & \\
\hline
\end{tabular}

Compton cloud is very close to equilibrium with the seed photon environment. The electron temperature of the Compton cloud differs by a factor of a few from the BB seed photon temperature. We also have to note that a single BB spectrum can be reproduced either with $A \ll 1$ (small coverage of the seed photon radiation by the Compton cloud) or with $A \gg 1$ (large coverage) and $\alpha \gg 1$ (inefficient Comptonisation). The free parameters of the BMC model (apart from the normalization $C_{N}$ ) are the $\mathrm{BB}$ colour temperature, $k T_{\mathrm{bb}}$, the spectral index $\alpha$ (photon index $\Gamma=\alpha+1)$ and $\log A$.

We can describe all the spectra studied in this paper in terms of bulk motion Comptonisation. The results of the fits of our INTEGRAL spectra with the BMC model are reported in Table 3. For GX 340+0, GX 349+2, GX 9+9 and GX 3+1 only $k T_{\mathrm{bb}}$ is well constrained. This is not surprising given that in their spectra only the thermal bump is unambiguously detected. The best-fit colour temperatures are about $2.5 \mathrm{keV}$ using COMPTT or BB models. GX $17+2$ and GX $13+1$ have similar best-fit $\Gamma$-values, even if not well constrained, whereas the slope of GX 5-1 is steeper but much better constrained than in GX $17+2$ and GX $13+1$. The upper value of $\Gamma$ reported for Cyg X-2 $(\$ 3)$ could be indicative of the presence of photon up-scattering (Comptonisation) of BB photons originated in the disc and NS surface. One can arrive to similar conclusions for GX 9+1 even if the effect seems to be less prominent there $(\Gamma \lesssim 4)$. For the two dim Atoll sources $\mathrm{H} 1608-522$ and $\mathrm{H} 1705-440$ we find that $\Gamma$ is well constrained while there is no real information on $k T_{\mathrm{bb}}$. This is not surprising given that in their spectra we do not see evidence of the thermal bump (described by the BB in the BMC model) and so it is impossible to infer either the BB colour-temperature or its relative contribution to the merging flux. In Sco X-1, the whole set of the best-fit parameters is well constrained, given that both thermal bump and the hard tail are detected with high statistical significance.

Since our analysis uses data starting from $20 \mathrm{keV}$, careful interpretation should be given to the origin of the BB seed photons found in Table $3\left(k T_{\mathrm{bb}} \sim 2.5 \mathrm{keV}\right)$. A broad band analysis of GX 17+2 and Cyg X-2 spectra (Farinelli, private communication) seems to suggest that, similarly to the BHC case, the seed photons for the BMC model are rather soft, with a temperature close to disk emission values, $\sim 0.4-0.6 \mathrm{keV}$ (Barret et al. 2000). A second Comptonisation component, COMPTT, is also needed with $\sim 2.5 \mathrm{keV}$ seed photons coming most likely from the
NS surface. In our spectra the disk component is too soft to be detected and the BMC model interprets the $\sim 2.5 \mathrm{keV}$ seed photons as due to the emission from non-efficiently up-scattered seed photons within the bulk flow. The energy sampling of our data does not allow us to produce a complete discussion about the full geometry of the sources and global BMC model parameters, beyond the scope of this article, and this caveat should be kept in mind in all we discussed above that remains, in any case, qualitatively valid.

\section{Discussion}

We have analysed IBIS/ISGRI available public data on a sample of twelve persistent LMXBs containing a neutron star. We focused our study on the average spectral behaviour of the sources and classified them in terms of spectral states. As shown by the light-curves we presented, the sources have some degree of variability, but the average spectrum is a representation of the sources above $20 \mathrm{keV}$ and enables the study of the global properties.

\subsection{A scenario for spectral evolution in NS LMXBs}

We observe three main spectral states: a very soft state with source spectra that can be well described by a single thermal Comptonisation component, an intermediate state (thermal Comptonisation plus power-law) and a low/hard state (single power-law). We have successfully studied these three spectral states in the frame of the Generic Comptonisation Model (BMC, TMK96). We present our scenario of the spectral evolution of NS LMXBs starting from the low/hard state.

The low/hard state is characterized by a low mass accretion rate in the disc. In this case the gravitational energy release in the disc is much smaller than the one in the optically thin outer boundary of the corona (Compton cloud). The coronal outer boundary is presumably related to the adjustment shock (Titarchuk \& Fiorito 2004). The corona completely covers the seed photon area (high $\log A$ ) and the emergent spectrum is a result of the up-scattering (Comptonisation) of the seed photons in the corona. At the very low level of accretion rate, the bulk motion is extremely weak and the emergent spectrum can be interpreted as pure thermal Comptonisation with no bulk signature (GX 354-0 in Fig. 4). The bulk motion effect increases with 
accretion rate and the power-law signature starts to be visible in the spectrum, the cut-off is moved at a higher energy than what expected from pure thermal Comptonisation (H 1608-522 in Fig. 4). In either case, one cannot see any trace of the seed photons in ISGRI (that give the BB bump). Open magnetic field lines are exposed to the observer but the outflow is weak because of the low mass accretion rate (there is not enough radiation in the disc to launch the wind) and the system is radio-quiet.

In the intermediate state the mass accretion rate increases with respect to the low/hard state. It leads to high efficiency of the Comptonisation, particularly bulk inflow Comptonisation that is seen as an extended hard tail in the spectrum. Thermal Comptonisation becomes less efficient because the coronal plasma is cooled down by the seed photons coming from the disc and NS surface. The corona consists of a quasi-spherical component (related to the closed field lines and bulk motion inflow) and of a cylindrical component (related to the open field lines and outflow, TS05). The vertical size of the cylindrical configuration is suppressed as the mass accretion increases. We start to see the seed blackbody bumps (only the higher energy one in the IBIS/ISGRI range), because the corona is cooled down and becomes more compact.

The very soft state is characterized by a high mass accretion rate that is very close to the critical (Eddington) values. The emergent spectrum is a sum of two blackbody-like spectra, one is related to the Comptonisation of the NS photons (visible in the IBIS/ISGRI range) and the other one is related to the Comptonisation of the disc photons. The seed photon and plasma temperatures differ by a factor of a few. The electron plasma and the photons in the Compton cloud are very close to equilibrium. In this state the corona is quasi-spherical, there is no bulk motion and no radio emission. In fact, the radiation pressure caused by the strong emission from the NS surface stops the bulk inflow and the high accretion rate changes the configuration of the field lines and the radio emission is quenched.

It is important to note that what plays the key role to suppress the bulk motion is the local radiation pressure, hence the local accretion rate impinging the bulk inflow: Sco X-1 has a total isotropically-estimated luminosity that is higher than e.g. GX $3+1$, but we speculate that the inflow anisotropy is much higher in GX $3+1$ than in Sco $X-1$, resulting in a higher local radiation pressure, i.e. bulk suppression.

\subsection{The radio emission - hard $X$-ray tail connection}

In the scenario proposed in the previous Section there is a clear connection between the X-ray spectral states and the radio emission: low/hard states are associated to weak radio emission, the radio emission increases in the intermediate state and then is quenched in the very soft state.

This trend is clearly met in the observations. We can imagine a continuous "accretion line" increasing from Atoll sources (island state to banana) moving to the $\mathrm{Z}$ sources (horizontal, normal and flaring branch). Only a few low-dim Atoll sources (typical low/hard state) have been detected in the radio band because of their low radio luminosity (Fender \& Hendry 2000; Migliari \& Fender 2006). Z sources in their horizontal branch have a spectrum that corresponds to the intermediate state we defined here and indeed a clear radio emission has been reported in this state (Fender \& Hendry 2000; Di Salvo \& Stella 2002, and references therein). The normal and flaring branch of $\mathrm{Z}$ sources corresponds to the very soft state defined here where no radio emission is expected and in fact $\mathrm{Z}$ sources in these branches have a severely reduced radio emission, if any.
Table 4. Comparison of the average radio $(8.5 \mathrm{GHz})$ and $\mathrm{X}$-ray hard tail (40-100 keV) flux of the $\mathrm{Z}$ and bright Atoll sources studied in this paper. The radio data are taken from Fender \& Hendry (2000) and Migliari \& Fender (2006).

\begin{tabular}{lcc}
\hline \hline Source & $\begin{array}{c}\text { Radio flux } \\
\text { (mJy) }\end{array}$ & $\begin{array}{c}\text { Hard tail flux } \\
10^{-11} \mathrm{erg} \mathrm{s}^{-1} \mathrm{~cm}^{-2}\end{array}$ \\
\hline Z sources & & \\
\hline Sco X-1 & $10 \pm 3$ & 18 \\
GX 17+2 & $1.0 \pm 0.3$ & 1.9 \\
GX 349+2 & $0.6 \pm 0.3$ & $<1.15$ \\
Cyg X-2 & $0.6 \pm 0.2$ & 1.7 \\
GX 5-1 & $1.3 \pm 0.3$ & 1.9 \\
GX 340+0 & $0.6 \pm 0.3$ & 2 \\
\hline Atolls & & \\
\hline GX 9+1 & $<0.2$ & $<0.75$ \\
GX 9+9 & $<0.2$ & $<1.72$ \\
GX 3+1 & $<0.3$ & $<0.43$ \\
GX 13+1 & $1.8 \pm 0.3$ & 1.74 \\
\hline
\end{tabular}

$\mathrm{Z}$ sources are much brighter radio emitters than Atolls and GX $13+1$ has an important radio emission, thus behaves like a Z (Fender \& Hendry 2000; Migliari \& Fender 2006). In average, unlike bright Atolls, $Z$ sources have a hard tail besides the thermal Comptonisation by an optically thick plasma of $\sim 3 \mathrm{keV}$ (Di Salvo \& Stella 2002, and this work); GX 13+1 has a hard tail (this work), thus again behaves like a Z. We basically see that in GX 13+1, the link radio emission - hard X-ray tail (both "against" its originally declared nature to be an Atoll source) reveals itself in a solid way.

Triggered by the GX $13+1$ case, we compared the average radio emission of all the sources of our sample with the hard $\mathrm{X}$-ray tail that appears on top of the $\sim 3 \mathrm{keV}$ Comptonisation spectrum. We note that the hard X-rays from the two soft X-ray dim Atoll sources, H 1608-522 and H 1705-440, are mainly coming from Comptonisation of soft photons in a hot corona (i.e. the overall spectrum is hard, $k T_{\mathrm{e}}>40 \mathrm{keV}$, see Table 2) and since we cannot disentangle the dominating thermal Comptonisation component from the dynamical (bulk) one, we do not include these two sources in the radio - hard tail study. Table 4 reports the average radio flux as derived by Fender \& Hendry (2000) and Migliari \& Fender (2006) and the 40-100 keV flux of the hard tails detected in this work. When a hard tail is detected, its $40-100 \mathrm{keV}$ flux is computed after fixing the COMPTT normalization to zero. For the sources where a hard tail was not detected, we computed $3 \sigma$ upper limits $(3 \times$ error in the $40-100 \mathrm{keV}$ range), assuming that all the flux in the $40-100 \mathrm{keV}$ comes from the hard tail. Figure 7 is visualising the relation between the average radio and hard tail (40-100 keV) luminosities computed using the distances in Table 1 . The correlation between the two is quite striking (Spearman rank order significance of $99.6 \%$ ) and the upper limits are consistent with the trend. We note that we obtain a very good correlation (Spearman rank order significance $99.2 \%$ ) also using the radio and hard tail fluxes instead of the luminosities. We chose to plot the luminosity correlation in order to show the intrinsic properties of the sources.

In Fig. 7 we have plotted the average radio and X-ray hard tail luminosities: the radio luminosities are the superposition of optically thick emission (compact jet) and optically thin flaring activity. The X-ray hard tail luminosities are the average of all hard tail states (from maximum to minimum strength). We are aware that these correlations are based on data that are not taken simultaneously, but they are all based on average flux that we can consider a good representation of the physics 


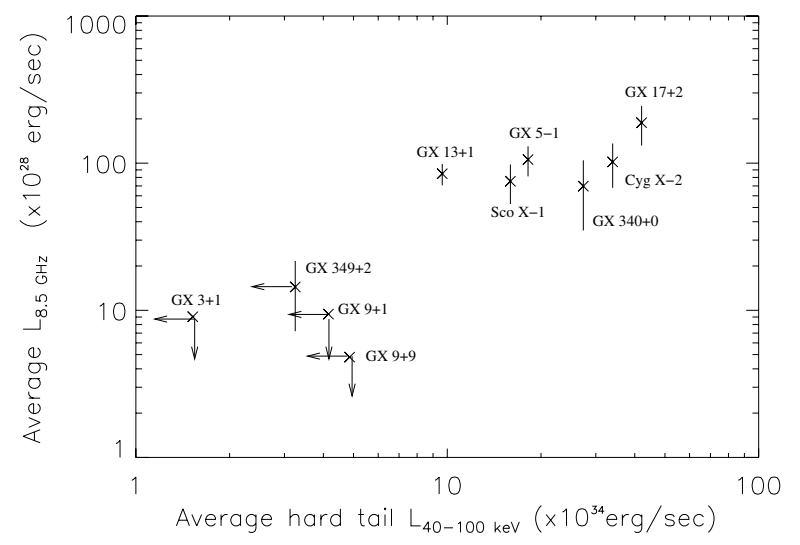

Fig. 7. Average radio luminosity plotted against the average hard X-ray tail (40-100 keV) luminosity. The correlation is clearly visible also if we compare the source fluxes instead of the luminosities (see text).

involved in these objects. For the sources where a hard tail has been observed, we compare the radio emission with what is left in the X-ray spectra of the sources once we remove the dominant Comptonisation component. In this respect, our detection of a hard tail in the spectrum of GX $13+1$ is important: GX $13+1$ has a similar X-ray flux (and luminosity) as the remaining bright Atoll sources (GX 3+1, GX 9+1, GX 9+9, see Table 1) but a much higher average radio and hard tail emission (Table 4), re-confirming the radio hard X-ray tail connection.

In the presence of a cut-off, the hard tail can be explained via Comptonisation of soft seed photons in the jet and/or in the corona. For the case where no cut-off is detected, many models have been proposed: Comptonisation by a hybrid (thermal nonthermal) corona (Coppi 1999), synchrotron emission from the electrons of the jet (Markoff et al. 2005) or bulk motion inflow (dynamical) Comptonisation (TMK96, used in this work).

The real test of any of these models can be done using the (variability) analysis of the power density spectrum (PDS) of the hard tail emission. The characteristic (break and QPO) frequencies of PDS do determine the geometric size of the configuration where the hard tail emission is formed. TS05 made this type of analysis for $4 \mathrm{U} 1728-34$ and found evidence that the hard tails are formed in the compact Compton cloud with geometry changing from cylindrical-like in the low/hard state to the quasispherical one in the high/soft state. Note that the X-ray-radio correlation along with the QPO-radio correlation is well established in 4U 1728-34. TS05 presented an explanation of these correlations in the framework of an oscillation model using the observed correlations of QPO low frequencies and their ratio.

The correlation we find between the radio and hard X-ray tail emission suggests that the hard tail formation area and the source of energetic electrons, ultimately causing the radio emission, are closely connected. The most probable site of this configuration is the NS magnetosphere. It can be suggested that the open magnetic field lines of the NS magnetosphere are the base of the jet seen in the radio emission. An increasing accretion rate leads to a more efficient radio emission (low/hard state to intermediate state) up to a point where the extremely high accretion rate (very soft state) changes the configuration of the field lines and the radio emission is quenched.

Acknowledgements. The authors thank A. Segreto and C. Ferrigno for the useful cross checks in the analysis phase and M. Falanga for the IBIS/ISGRI spectrum of GX 354-0. A.P. thanks M. R. Gaber, B. E. O'Neel and G. A. Wendt III for their precious contribution to the development of post-processing tools. A.P., A.B. and S.M. acknowledge the Italian Space Agency financial and programmatic support via contract I/R/046/04.

\section{References}

Asai, K., Dotani, T., Mitsuda, K., et al. 1994, PASJ, 46, 479

Barret, D. 2001, Adv. Space Res., 28, 307

Barret, D., \& Olive, J.-F. 2002, ApJ, 576, 391

Barret, D., Olive, J. F., Boirin, L., et al. 2000, ApJ, 533, 329

Christian, D. J., \& Swank, J. H. 1997, ApJS, 109, 177

Coppi, P. S. 1999, in ASP Conf. Ser. 161: High Energy Processes in Accreting Black Holes, ed. J. Poutanen, \& R. Svensson

D'Amico, F., Heindl, W. A., Rothschild, R. E., \& Gruber, D. E. 2001, ApJ, 547, L147

Di Salvo, T., \& Stella, L. 2002, in Proc. of the XXXVIIth Rencontres de Moriond, The Gamma-Ray Universe, ed. A. Goldwurm, D. N. Neumann, \& Jean Tran Thanh Van, 67

Di Salvo, T., Robba, N. R., Iaria, R., et al. 2001, ApJ, 554, 49

Di Salvo, T., Farinelli, R., Burderi, L., et al. 2002, A\&A, 386, 535

Di Salvo, T., Goldoni, P., Stella, L., et al. 2006, ApJ, submitted

Falanga, M., Gotz, D., Goldoni, P., et al. 2006, A\&A, 458, 21

Farinelli, R., Frontera, F., Zdziarski, A. A., et al. 2005, A\&A, 434, 25

Fender, R. P., \& Hendry, M. A. 2000, MNRAS, 317, 1

Ford, E. C., van der Klis, M., Méndez, M., et al. 2000, ApJ, 537, 368

Goldwurm, A., David, P., Foschini, L., et al. 2003, A\&A, 411, L223

Hasinger, G., \& van der Klis, M. 1989, A\&A, 225, 79

Homan, J., van der Klis, M., Wijnands, R., Vaughan, B., \& Kuulkers, E. 1998, ApJ, 499, L41

Homan, J., Wijnands, R., Rupen, M. P., et al. 2004, A\&A, 418, 255

Iaria, R., Di Salvo, T., Robba, N. R., et al. 2004, ApJ, 600, 358

Iaria, R., di Salvo, T., Robba, N. R., et al. 2005, A\&A, 439, 575

Kong, A. K. H., Charles, P. A., Homer, L., Kuulkers, E., \& O’Donoghue, D. 2006, MNRAS, 368, 781

Lavagetto, G., Iaria, R., di Salvo, T., et al. 2004, Nucl. Phys. B Proc. Suppl., 132, 616

Lebrun, F., Leray, J. P., Lavocat, P., et al. 2003, A\&A, 411, L141

Markoff, S., Nowak, M. A., \& Wilms, J. 2005, ApJ, 635, 1203

Migliari, S., \& Fender, R. P. 2006, MNRAS, 366, 79

Oosterbroek, T., Barret, D., Guainazzi, M., \& Ford, E. C. 2001, A\&A, 366, 138

Paizis, A., Ebisawa, K., Tikkanen, T., et al. 2005, A\&A, 443, 599

Poutanen, J., \& Svensson, R. 1996, ApJ, 470, 249

Rodriguez, J., Shaw, S. E., \& Corbel, S. 2006, A\&A, 451, 1045

Schulz, N. S., Hasinger, G., \& Truemper, J. 1989, A\&A, 225, 48

Shaposhnikov, N., \& Titarchuk, L. 2006, ApJ, 643, 1098

Sidoli, L., Paizis, A., Bazzano, A., \& Mereghetti, S. 2006, A\&A, [arXiv: astro-ph/0609221]

Titarchuk, L. 1994, ApJ, 434, 570

Titarchuk, L., \& Fiorito, R. 2004, ApJ, 612, 988

Titarchuk, L., \& Shaposhnikov, N. 2005, ApJ, 626, 298

Titarchuk, L., Mastichiadis, A., \& Kylafis, N. D. 1996, A\&AS, 120, 171

Titarchuk, L., Mastichiadis, A., \& Kylafis, N. D. 1997, ApJ, 487, 834

Ubertini, P., Lebrun, F., Di Cocco, G., et al. 2003, A\&A, 411, L131

Vilhu, O., Paizis, A., Hannikainen, D., et al. 2006, in prep.

Winkler, C., Courvoisier, T. J.-L., Di Cocco, G., et al. 2003, A\&A, 411, L1

Zhang, S. N., Harmon, B. A., Paciesas, W. S., et al. 1996, A\&AS, 120, 279 